\title{
Gain Measurement of Broadband Quantum Dot SOA by Two-Section Technique Yi-Shin Su ${ }^{a}$,Wei-Che Chang, Chao-Hsin Wu, and Ching-Fuh Lin ${ }^{a, b}$ \\ ${ }^{a}$ Institute of Electro-Optical Engineering, National Taiwan University, Taipei, Taiwan, R.O.C. \\ ${ }^{b}$ Department of Electrical Engineering and Institute of Electronics Engineering, National Taiwan University, Taipei, Taiwan, R.O.C. \\ Tel : 886-2-23635251-405 Fax : 886-2-23677467 \\ Email :cflin@cc.ee.ntu.edu.tw
}

The realization of lasers and optical amplifiers with self-organized quantum-dot materials has been of interest recently because QD devices have high differential gain, low threshold current, and improved temperature characteristics. In addition, the extremely low density of states per unit volume and adjustable dot size distribution makes QD devices suitable for broadband applications. Such a laser has been fabricated with operation current density lower than QW devices [1] for an order of magnitude. Our work is to obtain the actual gain profile of these broadband QD devices.

In this experiment, the active layer is grown on a GaAs substrate and has two types of InAs QD layers. One is for $1.24 \mu \mathrm{m}$ emitting wavelength. The other is for $1.28 \mu \mathrm{m}$ emitting wavelength. The QD layers is grown within InGaAs/GaAs QWs for better carrier confinement. The device used in the experiment has two section double channel ridge waveguide fabricated on the wafer by wet etch. To prevent reflection of light from the facet back into the waveguide, the waveguide direction is tilted from facet normal by 7 degrees, as shown in Fig. 1. The length of each section is $200 \mu \mathrm{m}$ and $800 \mu \mathrm{m}$, respectively. The EL spectrums from both side of this device are measured under the same current density. Figure 2 is the measured EL spectrum of the $800 \mu \mathrm{m}$ section. The spectrum has two peaks at $1.25 \mu \mathrm{m}$ and $1.17 \mu \mathrm{m}$. They correspond to the first quantized state and the second quantized state of the QDs. In addition, extra signals can be observed between $1100 \mathrm{~nm}$ and $1000 \mathrm{~nm}$ with $240 \mathrm{~mA}$ and $320 \mathrm{~mA}$ injection current. This is considered to be the contribution of QW states.

The EL spectrum from both sides of this device under the same injection density can be related by the following equation.

$$
\mathrm{S}_{2}\left(\mathrm{I}_{2}, \lambda\right)=\mathrm{S}_{1}\left(\mathrm{I}_{1}, \lambda\right) \times \exp \left[\mathrm{Gm}\left(\mathrm{I}_{1}, \mathrm{I}_{2}, \lambda\right) \times \mathrm{L} 2\right]
$$

In the equation, $S_{2}\left(I_{2}, \lambda\right)$ is emission spectrum of $800 \mu m$ section, and $S_{1}\left(I_{1}, \lambda\right)$ emission spectrum of $200 \mu m$ section. L2 is the waveguide length difference between the two sections. Because the two sections are fabricated on the same chip, the variation of material gain and spontaneous emission spectrum should be much smaller than devices fabricated on different chips. Thus, the measured spectrum of 200 um section can be considered as the spectrum of lights propagating at " $\mathrm{A}$ " point of the $800 \mathrm{um}$ section toward the facet of 800 um section (Figure 1). Thus the light measured at the facet of 800 um section is the stimulated emission of light propagating from point $\mathrm{A}$. The gain spectrum can be calculated by dividing the emission spectra of 800 um section and 200un section. Figure 3 is the obtained gain spectrum of the $800 \mathrm{um}$ section. There are two gain peaks at $1230 \mathrm{~nm}$ and $1140 \mathrm{~nm}$. The maximum gain at $1230 \mathrm{~nm}$ is $30.8 \mathrm{~cm}^{-1}$. This peak already saturates for injection current below $80 \mathrm{~mA}$. The gain at the $1140 \mathrm{~nm}$ peak saturates at a higher injection level, and can be increased up to $42 \mathrm{~cm}^{-1}$. At the same time, the contribution of the QW layers to the gain appears between $1050 \mathrm{~nm}$ and $1100 \mathrm{~nm}$. More details of the measured gain spectrum will be

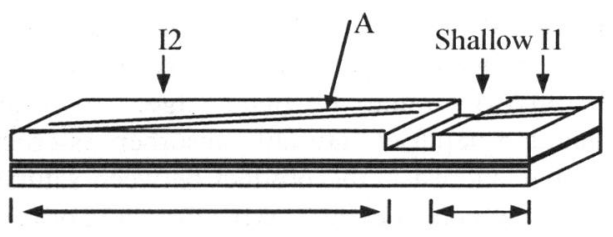

L2

L1

Fig. 1 The Device Structure

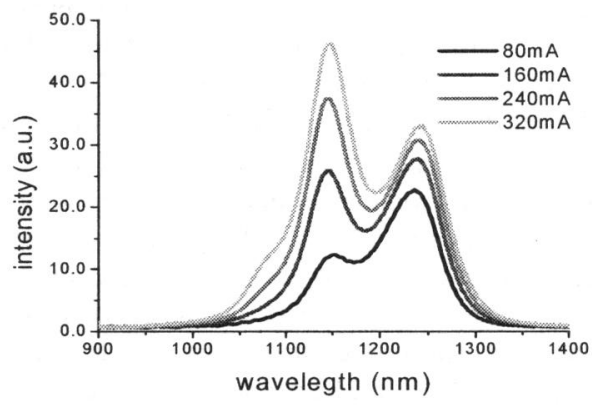

Fig. 2. EL Spectrum of the 800um

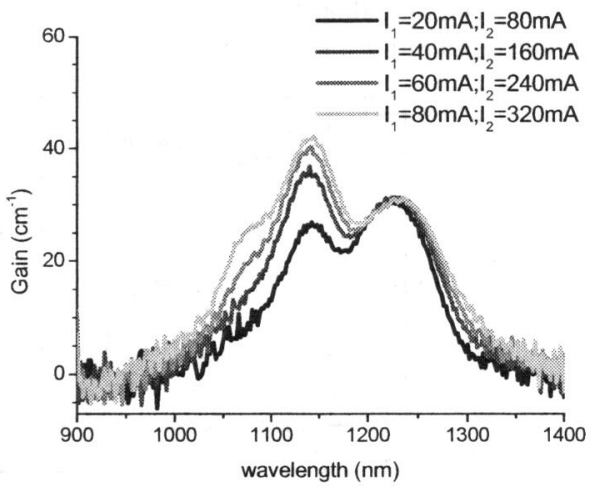

Fig. 3. Gain presented.

References:

[1] P.M. Varangis, H. Li, G.T. Liu, T.C. Newell, A. Stintz, B. Fuchs, K.J. Malloy and L.F. Lester, "Low-threshold quantum dot lasers with $201 \mathrm{~nm}$ tuning range," Electron. Lett., 2000, Vol. 36, Nov. 18 